\title{
Nota Científica \\ Primera estimación de la tasa de fijación biológica de nitrógeno en Cuenca Alfonso, región sur del Golfo de California
}

\author{
First estimation of biological nitrogen fixation rate in Alfonso Basin, \\ southern Gulf of California
}

\section{Cristian Hakspiel-Segura ${ }^{1}$ y Aída Martínez-López ${ }^{1}$}

\begin{abstract}
${ }^{1}$ Centro Interdisciplinario de Ciencias Marinas-IPN (CICIMAR), Av. Instituto Politécnico Nacional s/n, Col. Playa Palo de Santa Rita, La Paz, B.C.S. 23096, México. amartin@ipn.mx

Abstract.- Nitrogen-fixation (FBN) rates were determined for the first time in the euphotic zone $(0-43 \mathrm{~m})$ and at $150 \mathrm{~m}$ depth using the high-sensitive ${ }^{15} \mathrm{~N}_{2}$ tracer method at Alfonso Basin, Gulf of California, during May 2012. In this study, high rates


of hypoxic conditions relative to other observations restricted to the euphotic zone in systems of the Gulf and the Eastern North Pacific. These data support previous affirmations about the significant FBN regulation pathway on the nitrogen inventory in both, photic and aphotic zone.
\end{abstract}

Key words: Nitrogen fixation, hypoxic conditions, Alfonso Basin

\section{INTRODUCCIÓN}

La fijación biológica de nitrógeno (FBN) consiste en la reducción de gas $\mathrm{N}_{2}$ a una molécula biológicamente disponible para plantas y microorganismos en forma de amonio, el cual es luego asimilado a moléculas orgánicas (Capone \& Carpenter 1999). El nitrógeno, por su parte, se considera uno de los principales elementos reguladores de la producción primaria en los estratos superficiales y subsuperficiales de la mayoría de los sistemas marinos. Históricamente, esta actividad ha sido subestimada, sin embargo la FBN, en la actualidad se reconoce como un aporte relevante de nitrógeno biodisponible que podría solventar en gran medida esta condición limitante (Vitousek \& Howarth 1991). Asimismo, estudios recientes han demostrado que sus tasas puede ser igual o incluso mayores que las estimadas para ambientes terrestres (Ward et al. 2007). Actualmente, algunas modelaciones globales y regionales basadas en trazadores biogeoquímicos han permitido dilucidar la magnitud de la FBN y otros procesos dentro del inventario del nitrógeno del océano. No obstante, dichas estimaciones se basan en supuestos que requieren de verificación por medio de cuantificaciones directas, las cuales hoy en día comienzan a ser compiladas en bases datos y comparadas de acuerdo a las metodologías empleadas (Großkopf et al. 2012, Luo et al. 2012).

En el océano, la FBN puede ser realizada por una diversidad de organismos entre los que se encuentran los diazótrofos (aquellos que son capaces de fijar nitrógeno molecular), que hasta hace pocos años se consideraba principalmente a las cianobacterias en sus diferentes formas; filamentosas (heterocísticas y no heterocísticas), unicelulares y simbióticas (ver referencias en Berman-Frank et al. 2003). Sin embargo, en años recientes se ha comprobado que las bacterias heterótrofas y arqueas también pueden realizar esta función (Zehr et al. 2001, Biegala \& Raimbault 2008, Langlois et al. 2008, Bonnet et al. 2013). A nivel internacional aún existen muchos vacíos de información no solo de las tasas de FBN, sino también de la diversidad de organismos participantes en la introducción de nitrógeno nuevo al océano, particularmente para algunas regiones en el mundo de gran interés científico por sus niveles de producción y características oceanográficas como la confluencia de condiciones óxicas y de mínimo oxígeno en la columna de agua, como es el caso de Cuenca Alfonso en la Bahía de La Paz (Golfo de California). Este sitio, consolidado desde el 2002 como un observatorio de la variabilidad ambiental, producción biológica y del flujo de material sedimentario al fondo marino, ha permitido conocer algunos aspectos sobre la dinámica estacional de nutrientes y el ciclo de producción biológica. Sin embargo, la cuantificación de ciertos procesos como la FBN aún son desconocidos.

El presente estudio incluye la primera cuantificación del aporte de nitrógeno por FBN a la zona eufótica de 
Cuenca Alfonso durante mayo de 2013, así como una primera estimación para el Golfo de California a $150 \mathrm{~m}$ de profundidad. Parte de este trabajo probó la hipótesis de la ocurrencia de FBN durante la prevalencia de un enriquecimiento de nutrientes en subsuperficie, el cual es atribuido a una somerización de las isopicnas por la actividad de un giro ciclónico de submesoescala que se presenta estacionalmente entre marzo y julio (VillegasAguilera 2009, Martínez-López et al. 2012).

\section{Materiales y MÉTOdos}

Las muestras fueron colectadas en mayo de 2012 con botella Niskin en 5 profundidades, 4 de ellas en la zona eufótica $\left(100,33,3\right.$ y $\left.0,1 \% \mathrm{E}_{0}\right)$ y una a $150 \mathrm{~m}$ de profundidad, en Cuenca Alfonso (Fig. 1). Se llenaron 2 botellas de policarbonato transparente de $2 \mathrm{~L}$ (Nalgen) por cada profundidad y una de ellas se inoculó con gas ${ }^{15} \mathrm{~N}_{2}(98$ atom. $\% \mathrm{~N}_{2}$ ) como trazador, y la otra se utilizó como blanco. La incubación in situ se realizó por aproximadamente $24 \mathrm{~h}$, con el fin de facilitar la completa disolución del ${ }^{15} \mathrm{~N}_{2}$ inoculado y minimizar la subestimación que ha sido previamente reportada por Mohr et al. (2010) y Wilson et al. (2012) en la estimación de las tasas. El material particulado procedente de las muestras incubadas se analizó por espectrometría de masas de razones isotópicas en el laboratorio de isótopos estables (SIF) de la Universidad de California, Davis, para conocer el contenido elemental de nitrógeno y el enriquecimiento isotópico. Los procedimientos mencionados y los cálculos para la estimación de las tasas se realizaron de acuerdo a la metodología propuesta por Montoya et al. (1996).

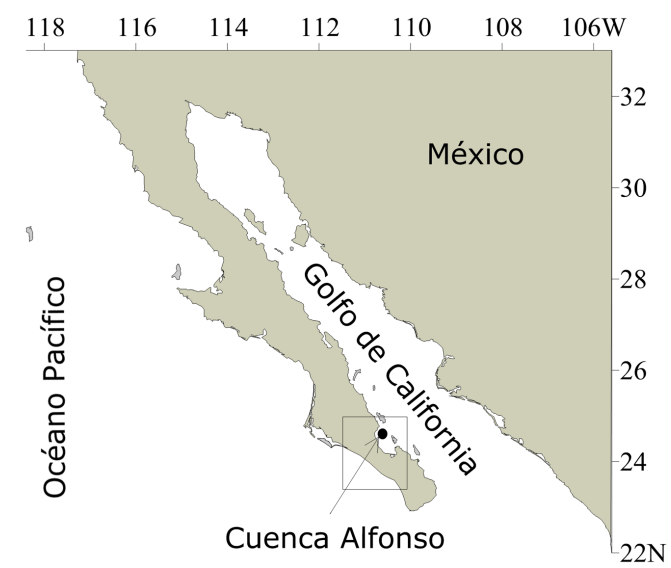

Figura 1. Localización del sitio de estudio en Cuenca Alfonso, Bahía de La Paz, México / Location of the study area in Alfonso Basin Bahía de La Paz, México
Complementariamente, con el propósito de cuantificar a los componentes planctónicos $(<20 \mu \mathrm{m})$ presentes en el sitio de estudio, se colectaron muestras para recuentos de nanoplancton autótrofo (cianobacterias y flagelados) y picoplancton (autótrofo y bacterias heterótrofas) las cuales fueron analizadas por técnicas estándar de microscopía de epifluorescencia y citometría de flujo, respectivamente. Para este último análisis, se utilizó un citómetro de flujo Beckman-Coulter Altra (operado por SOEST Citometría de Flujo) ${ }^{1}$.

\section{Resultados Y Discusión}

La asimilación de ${ }^{15} \mathrm{~N}_{2}$ junto con el ensayo de reducción de acetileno, han sido desde la década de los 60's las metodologías estándar más usadas y precisas para la determinación de las tasas de FBN (Luo et al. 2012). A diferencia de la primera esta última, es un método indirecto que determina la actividad de la nitrogenasa y cuya gran desventaja radica en la falta de exactitud en los factores usados para la conversión del etileno producido a $\mathrm{N}_{2}$ reducido en amonio, asociado a la variabilidad fisiológica de los organismos diazotróficos participantes y de las condiciones ambientales (Montoya et al. 1996, Zehr \& Montoya 2007). En el presente trabajo, el procedimiento de asimilación de ${ }^{15} \mathrm{~N}_{2}$ se efectuó a través de la inyección directa del gas al contenedor con la muestra de agua. De acuerdo a evaluaciones metodológicas realizadas por Mohr et al. (2010) y Wilson et al. (2012) este procedimiento es propenso de significativas subestimaciones en comparación con recientes cuantificaciones que consisten en la inoculación del gas ${ }^{15} \mathrm{~N}_{2}$ previamente disuelto en agua de mar filtrada y degasificada para incrementar su solubilidad. No obstante, en este trabajo con el propósito de minimizar este sesgo se incubó por un periodo de $\sim 24 \mathrm{~h}$, tiempo en el cual incubaciones in situ realizadas para Crocosphaera watsonii reportan que las tasas de FBN representan el $\sim 80 \%$ de la tasa máxima estimada a través del procedimiento alterno.

Las tasas instantáneas de FBN obtenidas en Cuenca Alfonso oscilaron entre 1,49-3,42 $\mathrm{nmol} \mathrm{N} \mathrm{L} \mathrm{d}^{-1}$, con valores relativamente poco fluctuantes en las 4 profundidades más superficiales correspondientes a la zona eufótica (100, 33, 3, 0,1 \% $\left.\mathrm{E}_{0}\right)$ (Fig. 2A). De acuerdo a estos niveles de actividad, la columna de agua presentó un valor integrado para la zona eufótica de $121 \mu \mathrm{mol} \mathrm{N} \mathrm{m}{ }^{-2}$ $\mathrm{d}^{-1}$, el cual se considera un valor de bajo a moderado con

${ }^{1}<$ www. soest.hawaii.edu/sfcf $>$ 
respecto a los rangos conocidos para el Golfo de California $\left(20-795 \mu \mathrm{mol} \mathrm{N} \mathrm{m}{ }^{-2} \mathrm{~d}^{-1}\right.$; media $\left.=214 \mu \mathrm{mol} \mathrm{N} \mathrm{m}{ }^{-2} \mathrm{~d}^{-1}\right)$ y el Pacífico Oriental Tropical ( $\left.20-23^{\circ} \mathrm{N}, 110^{\circ} \mathrm{W}\right)(14-233 \mu \mathrm{mol}$ $\mathrm{N} \mathrm{m}^{-2} \mathrm{~d}^{-1}$; media $\left.=86 \mu \mathrm{mol} \mathrm{N} \mathrm{m}^{-2} \mathrm{~d}^{-1}\right)$, los cuales fueron estimados únicamente para el periodo de verano y en muy bajas concentraciones de $\mathrm{NO}_{2}^{-}+\mathrm{NO}_{3}^{-}(<0,1 \mu \mathrm{M})$ en la columna superficial de agua (White et al. 2007, 2012).

$\mathrm{El}$ valor registrado a $150 \mathrm{~m}$ fue menor a las mediciones de FBN en la zona eufótica (Fig. 2). Basados en observaciones complementarias, esta disminución en Cuenca Alfonso podría ser resultado de una leve inhibición del mecanismo fisiológico de la nitrogenasa (enzima que cataliza la FBN) causada por la incidencia de altas concentraciones de nitrógeno inorgánico $(8,11 \mu \mathrm{M}$ de $\mathrm{NO}_{2}^{-}+\mathrm{NO}_{3}^{-}$), y/o por la asociación de esta actividad de FBN a la densidad de los diazótrofos. A pesar de la baja tasa en el estrato profundo, su contribución incrementó de manera significativa la estimación del valor integrado de FBN en zona eufótica en 3,6 veces (437 $\mu \mathrm{mol}$ $\left.\mathrm{N} \mathrm{m}^{-2} \mathrm{~d}^{-1}\right)$. La existencia de FBN en profundidades mayores a la zona eufótica es aún pobremente comprendida. Al presente, solo se encuentran publicadas, algunas mediciones para la estación de monitoreo SPOTS (por su siglas en ingles San Pedro Ocean Time Series) en la Bahía del Sur de California de California $\left(\sim 33^{\circ} \mathrm{N}\right)$ entre 500-885 m (Hamersley et al. 2011), para la zona de mínimo oxígeno en el sistema de surgencia de Perú-Chile entre 120-400 m (Fernández et al. 2011) y para el Pacífico Suroriental Tropical entre el límite superior de la zona afótica hasta $2000 \mathrm{~m}$ (Bonnet et al. 2013), los cuales han detectado tasas integradas de 55, $574 \pm 294$ y $24-509 \mu \mathrm{mol}$ $\mathrm{N} \mathrm{m}^{-2} \mathrm{~d}^{-1}$, respectivamente. Estas observaciones corrobaran al igual que nuestros resultados, que la actividad diazotrófa observada por debajo del $0,1 \% \mathrm{E}_{0}$, en algunas ocasiones, es superior por varias veces a la que ocurre en la zona eufótica. Estos últimos hallazgos de manera particular han abierto el debate sobre la incidencia de esta actividad en ambientes en donde hay considerables niveles de nitrógeno inorgánico disuelto (principalmente en forma de nitrato) y a su vez ocurren intensos procesos de remoción (desnitrificación y/o ANAMMOX) de nitrógeno (Landolfi et al. 2013).

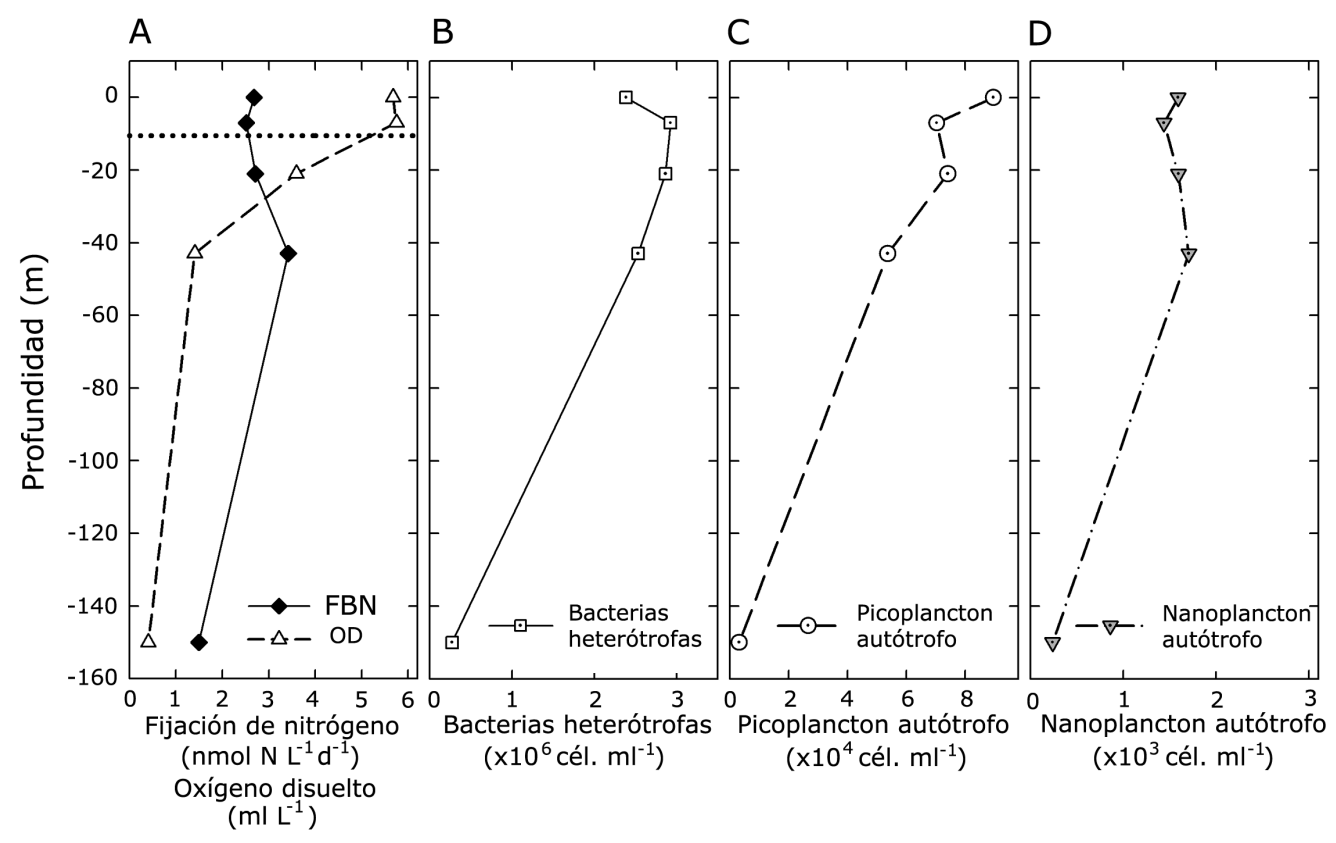

Figura 2. Distribución vertical de: (A) tasas de fijación biológica de nitrógeno (FBN); (B) bacterias heterotróficas; (C) picoplancton autótrofo y (D) nanoplancton autótrofo durante mayo de 2012 en Cuenca Alfonso. La línea punteada en (A) corresponde a la profundidad de la capa de mezcla / Vertical distribution of: (A) biological nitrogen fixation (BNF) rates; (B) heterotrophic bacteria; (C) autotrophic picoplankton and (D) autotrophic nanoplankton during May 2012 in Cuenca Alfonso. The dotted line in (A) corresponds to the depth of the mixed layer 
En este estudio, a diferencia de otros meses, durante mayo fue evidente la presencia de una capa con niveles hipóxicos $\left(1,4 \mathrm{ml} \mathrm{L}^{-1}\right)$ por debajo de $50 \mathrm{~m}$ en Cuenca Alfonso (Fig. 2A). No hay claridad si esta condición fue producto del posible bombeo estacional de aguas más profundas a través del giro ciclónico, o al abatimiento de oxígeno inducido por la degradación del material orgánico en hundimiento que fue producido por el ingreso de nutrientes a la zona eufótica, o simplemente una combinación de ambos procesos. Sin embargo, la expansión de esta capa de bajo oxígeno parecería incrementar las posibilidades del acoplamiento espacial entre la FBN y los procesos de pérdida de nitrógeno, de manera que la FBN resultaría ser un mecanismo de retroalimentación positiva para balancear el inventario de nitrógeno (Landolfi et al. 2013).

De acuerdo a los perfiles verticales de los componentes planctónicos en la Cuenca Alfonso, la distribución de los componentes $<2 \mu \mathrm{m}$ no fue substancialmente diferente a la variabilidad de la tasa de FBN, de manera que no se descarta la posibilidad de que su contribución a esta actividad pueda ser relevante (Fig. 2B, C); sin embargo, la abundancia de nanoplancton autótrofo mostró la mayor semejanza con esta actividad (Fig. 2D). Esto es consistente con otras mediciones de tasas de FBN fraccionadas por tamaños y la expresión de genes nifH (gen estructural que codifica el complejo de la enzima nitrogenasa), las cuales revelan que esta actividad en algunos sistemas del Pacífico Norte se encuentra dominada principalmente por cianobacterias cocoides $<10 \mu \mathrm{m}$, fracción que incluye a los representantes del pico y el nanoplancton (Church et al. 2008, Hamersley et al. 2011). No obstante estos indicios, en el futuro otros estudios son necesarios para concretar conclusiones sobre la identidad de los microorganismos participantes, en esta ruta del nitrógeno.

Las condiciones ambientales de este trabajo difieren substancialmente a las de los reportes previos de tasas de FBN para aguas abiertas de la parte sur y central del Golfo de California durante el verano (White et al. 2007, 2012), cuando predomina una columna de agua estratificada y baja concentración de nutrientes inorgánicos; aun así los niveles de FBN observados aquí fueron comparables. La detección de tasas de FBN relativamente moderadas durante mayo de 2012 sugieren que en Bahía de La Paz, esta ruta constituye una entrada importante de este elemento al sistema incluso bajo altas concentraciones de nutrientes inorgánicos nitrogenados (1,78-20,0 $\mu \mathrm{M})$. No obstante, más determinaciones deben efectuarse para corroborar la hipótesis de que la magnitud de esta actividad, en un ciclo anual, posiblemente esté siendo regulada por la concentración de nutrientes inorgánicos, así como por la dinámica de las poblaciones diazotróficas en la columna de agua. Para esto último, identificaciones más precisas de los potenciales fijadores de nitrógeno deben ser realizadas, para lo cual es recomendable la realización de análisis genómicos, proteómicos y/o transcriptómicos que permitan asociar su identidad y actividad en la columna de agua.

\section{Agradecimientos}

Al Instituto Politécnico Nacional por el apoyo recibido a través del proyecto SIP (20101059), por los estímulos recibidos por AML de la Comisión de Operación y Fomento de Actividades Académicas (COFAA-IPN) y Estímulos al Desempeño en Investigación (EDI) y la beca PIFI recibida por CHS. Al CONACYT por la beca recibida por CHS. Agradecemos al Dr. Douglas G. Capone por la capacitación teórica en la técnica de asimilación de ${ }^{15} \mathrm{~N}_{2}$ en el marco del taller NITROPAL efectuado en el CICIMAR-IPN; a Troy Gunderson por la capacitación técnica en la Universidad del Sur de California (USC).

\section{LITERATURA CITADA}

Bonnet S, J Dekaezemacker, KA Turk-Kubo, T Moutin, RM Hamersley, O Grosso1, JP Zehr \& DG Capone. 2013. Aphotic $\mathrm{N}_{2}$ fixation in the Eastern Tropical South Pacific Ocean. PLoS ONE 8(12): e81265 <doi:10.1371/ journal.pone.0081265>

Berman-Frank I, P Lundgren \& P Falkowski. 2003. Nitrogen fixation and photosynthetic oxygen evolution in cyanobacteria. Research in Microbiology 154: 157-164.

Biegala IC \& P Raimbault. 2008. High abundance of diazotrophic picocyanobacteria $(<3 \mu \mathrm{m})$ in a Southwest Pacific coral lagoon. Aquatic Microbial Ecology 51: 45-53.

Capone DG \& EJ Carpenter. 1999. Nitrogen fixation by marine cyanobacteria: historical and global perspectives. In: Charpy L \& AWD Larkum (eds). Bulletin de l'Institut Océanographique, Mónaco, Special Issue 19: 235-236.

Church MJ, KM Björkman, DM Karl, MA Saito \& JP Zehr. 2008. Regional distributions of nitrogen-fixing bacteria in the Pacific Ocean. Limnology and Oceanography 53: 63-77.

Fernández C, L Farías \& O Ulloa. 2011. Nitrogen fixation in denitrified marine waters. PLoS ONE 6(6): e20539 <doi:10.1371/journal.pone.0020539>

Großkopf T, W Mohr, T Baustian, H Schunck, D Gill, MM Kuypers, G Lavik, RA Schmitz, DWR Wallace \& J LaRoche. 2012. Doubling of marine dinitrogen-fixation rates based on direct measurements. Nature 488: 361-364. 
Hamersley MR, KA Turk, A Leinweber, N Gruber, JP Zehr, T Gunderson \& DG Capone. 2011. Nitrogen fixation within the water column associated with two hypoxic basins in the Southern California Bight. Aquatic Microbial Ecology 63: 193-205.

Landolfi A, H Dietze, W Koeve \& A Oschlies. 2013. Overlooked runaway feedback in the marine nitrogen cycle: the vicious cycle. Biogeosciences 10: 1351-1363.

Langlois RJ, D Hummer \& J LaRoche. 2008. Abundances and distributions of the dominant nifH phylotypes in the Northern Atlantic Ocean. Applied and Environmental Microbiology 74: 1922-1931.

Luo YW, SC Doney, S Bonnet, KH Boström, D Böttjer, DG Capone, EJ Carpenter, YL Chen, MJ Church, LI Falcón, RA Foster, K Furuya, K Gundersen, S Kitajima, RJ Langlois, J LaRoche, RM Letelier, PH Moisander, CM Moore, MR Mulholland, JA Needoba, KM Orcutt, AJ Poulton, P Raimbault, AP Rees, L Riemann, T Shiozaki, A Subramaniam, T Tyrrell, KA Turk, AE White \& JP Zehr. 2012. Database for Diazotrophs in global ocean: biomasses, nifH copies and nitrogen fixation rates. Earth System Science Data 4: 47-73 <doi:10.5194/essd-4-472012>

Martínez-López A, IG Álvarez-Gómez \& R Durazo. 2012. Climate variability and silicoflagellate fluxes in Alfonso Basin (Southern Gulf of California). Botanica Marina 55(2): 177-185.

Mohr W, T Großkopf, DWR Wallace \& J LaRoche. 2010. Methodological underestimation of oceanic nitrogen fixation rates. PLoS ONE 5(9): e12583. <doi:10.1371/ journal.pone.0012583>

Montoya JP, M Voss, P Kahler \& DG Capone. 1996. A simple, high-precision, high sensitivity tracer assay for $\mathrm{N}_{2}$ fixation. Applied and Environmental Microbiology 62: 986-993.
Villegas-Aguilera MM. 2009. Fitoplancton silíceo de la zona eufótica como señal de la productividad primaria en Cuenca Alfonso, Golfo de California. 2009. Tesis de Maestría. Centro Interdisciplinario de Ciencias Marinas-IPN, La Paz, 80 pp. <http://tesis.bnct.ipn.mx/dspace/bitstream/ 123456789/8128/1/FITOPLAC.pdf>

Vitousek PM \& RW Howarth. 1991. Nitrogen limitation on land and in the sea: How can it occur? Biogeochemistry 13: $87-115$.

Ward BB, DG Capone \& JP Zehr. 2007. What's new in the nitrogen cycle? Oceanography 20: 101-109.

White AE, FG Prahl, RM Letelier \& BN Popp. 2007. Summer surface waters in the Gulf of California: Prime habitat for biological $\mathrm{N}_{2}$ fixation. Global Biogeochemical Cycles 21: GB2017. <doi:10.1029/2006GB002779>

White AE, RA Foster, CR Benitez-Nelson, P Masqué, E Verdeny, BN Popp, KE Arthur \& FG Prahl. 2012. Nitrogen fixation in the Gulf of California and the Eastern Tropical North Pacific, Progress in Oceanography 109: 117 <dx.doi.org/10.1016/j.pocean.2012.09.002>

Wilson ST, D Böttjer, MJ Church \& DM Karl. 2012. Comparative assessment of nitrogen fixation methodologies conducted in the oligotrophic North Pacific Ocean. Applied Environmental Microbiology 78: 6491-6498.

Zehr JP \& JP Montoya. 2007. Measuring $\mathrm{N}_{2}$ fixation in the field. In: Bothe H, S Ferguson \& WE Newton (eds). Biology of the nitrogen cycle, pp. 193-205. Elsevier, Amsterdam.

Zehr JP, JB Waterbury, PJ Turner, JP Montoya, E Omoregie, GF Steward, A Hansen \& DM Karl. 2001. Unicellular cyanobacteria fix $\mathrm{N}_{2}$ in the subtropical North Pacific Ocean. Nature 412: 635-638. 